\title{
Metachronous Isolated Contralateral Lung Metastasis from Pulmonary Adenosquamous Carcinoma with EGFR Mutation
}

\author{
Hitomi Kawai ${ }^{1}$, Kesato Iguchi², Norio Takayashiki ${ }^{1}$, Shinichiro Okauchi ${ }^{3}$, Hiroaki Satoh ${ }^{3, *}$
}

\begin{abstract}
Lung metastasis and metachronous double primary lung cancer are both common and often present diagnostic challenges. We present a case of metachronous isolated contralateral lung metastasis from pulmonary adenosquamous carcinoma with EGFR mutation. A 75-yearold woman presented with left lung nodule on a routine follow-up chest radiograph. She had had surgery for pulmonary adenocarcinoma with EGFR Ex21 L858R mutation 6 years ago. She underwent surgical resection, and histologic findings revealed adenosquamous carcinoma with the same EGFR mutation. Re-assessment of the resected specimen of the primary tumor resected 6 years ago revealed the morphologically similarity to the left lung tumor. Based on morphological and genetic identity, final diagnosis was adenosquamous cell carcinoma and metachronous isolated contralateral lung metastasis. The diagnosis of metachronous isolated metastasis is difficult but important for appropriate management and prediction of prognosis. A careful pathological examination and evaluation of genetic abnormality are needed to make the correct diagnosis.
\end{abstract}

\section{KEYWORDS}

EGFR mutation; recurrence; lung adenosquamous carcinoma

AUTHOR AFFILIATIONS

${ }^{1}$ Division of Pathology, Mito Medical Center, University of Tsukuba-Mito Medical Center, Mito, Ibaraki, Japan

2 Division of Surgery, Mito Medical Center, University of Tsukuba-Mito Medical Center, Mito, Ibaraki, Japan

${ }^{3}$ Division of Respiratory Medicine, Mito Medical Center, University of Tsukuba-Mito Kyodo General Hospital, Mito, Ibaraki, Japan

* Corresponding author: Division of Respiratory Medicine, Mito Medical Center, University of Tsukuba, Miya-machi 3-2-7, Mito, Ibaraki, 310-0015, Japan; e-mail: hirosato@md.tsukuba.ac.jp

Received: 4 June 2019

Accepted: 20 June 2020

Published online: 1 October 2020

Acta Medica (Hradec Králové) 2020; 63(3): 141-144

https://doi.org/10.14712/18059694.2020.33

(c) 2020 The Authors. This is an open-access article distributed under the terms of the Creative Commons Attribution License (http://creativecommons.org/licenses/by/4.0), which permits unrestricted use, distribution, and reproduction in any medium, provided the original author and source are credited. 


\section{INTRODUCTION}

Metachronous isolated contralateral lung metastasis from pulmonary carcinoma is not rare. However, establishing diagnosis of intrapulmonary metastasis or is not necessarily easy. The metastatic tumors cannot entirely be the same as the primary tumor morphologically and lead to misdiagnosis. Such misdiagnosis lead to the inappropriate treatment and worse prognosis if each clinical condition differs significantly. On the other hand, there are patients whose second cancer histologically resembles the first cancer. In these patients, we must be aware of mistakes in selection of treatment. Although the EGFR mutation is one of the most common gene mutations, there has been only one published case report which showed the important role of the EGFR mutation in diagnosis of metachronous isolated contralateral lung metastasis intrapulmonary metastasis or second primary lung adenocarcinoma (1).

In comparison with adenocarcinoma and squamous cell carcinoma of the lung, adenosquamous cell carcinoma is an uncommon tumor with distinct clinical behavior $(2,3)$. There have been many reports that patients with adenosquamous cell lung cancer have poorer prognosis than

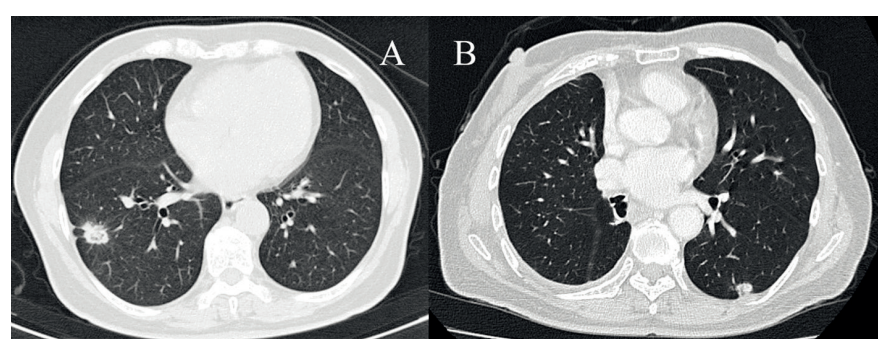

Fig. 1 Chest CT findings of the first tumor of the right lung $(A)$ and the second tumor of the left lung (B). those with lung adenocarcinoma and those with squamous cell lung cancer $(2,3)$. We report a prolonged survival case of a metachronous isolated contralateral lung metastasis from pulmonary adenosquamous carcinoma with EGFR mutation, which was evaluated as second primary lung cancer at the time of surgical resection.

\section{CASE REPORT}

A 69-year-old woman was admitted to our hospital due to an abnormal opacity in chest radiograph during mass-screening. She was a housewife and she never smoked. Chest CT scan revealed a nodule in the lower lobe of the right lung (Figure 1A). Hilar and mediastinal lymph node adenopathy was not observed. She was referred to our hospital for further examination and treatment. Physical examination and laboratory data were unremarkable. Right lower lobectomy with nodal dissection was performed, and the final pathological diagnosis was adenocarcinoma. Pathological tumor stage was proven to be IB. Mutation analysis of the tumor expressed epidermal growth factor receptor (EGFR) Exon 21 L858R mutation. She received postoperative adjuvant treatment with TS-1 for 5 years. Thereafter, she was followed up at our outpatient department without any additional adjuvant therapy. Six years after the surgery, a small nodule in the left lower lobe was detected on the chest CT scan (Figure 1-B). She received surgical resection to diagnose if this lesion was de novo tumor or possibly recurrence of lung cancer. Video-assisted thoracic surgery revealed a small nodule in the left lower lobe. Tumor size was $15 \times 7 \times 7 \mathrm{~mm}$ and surgical margin was free to the pleura. The component of the acinar type adenocarcinoma that proliferates in the glandular duct was main, but squamous cell carcinoma
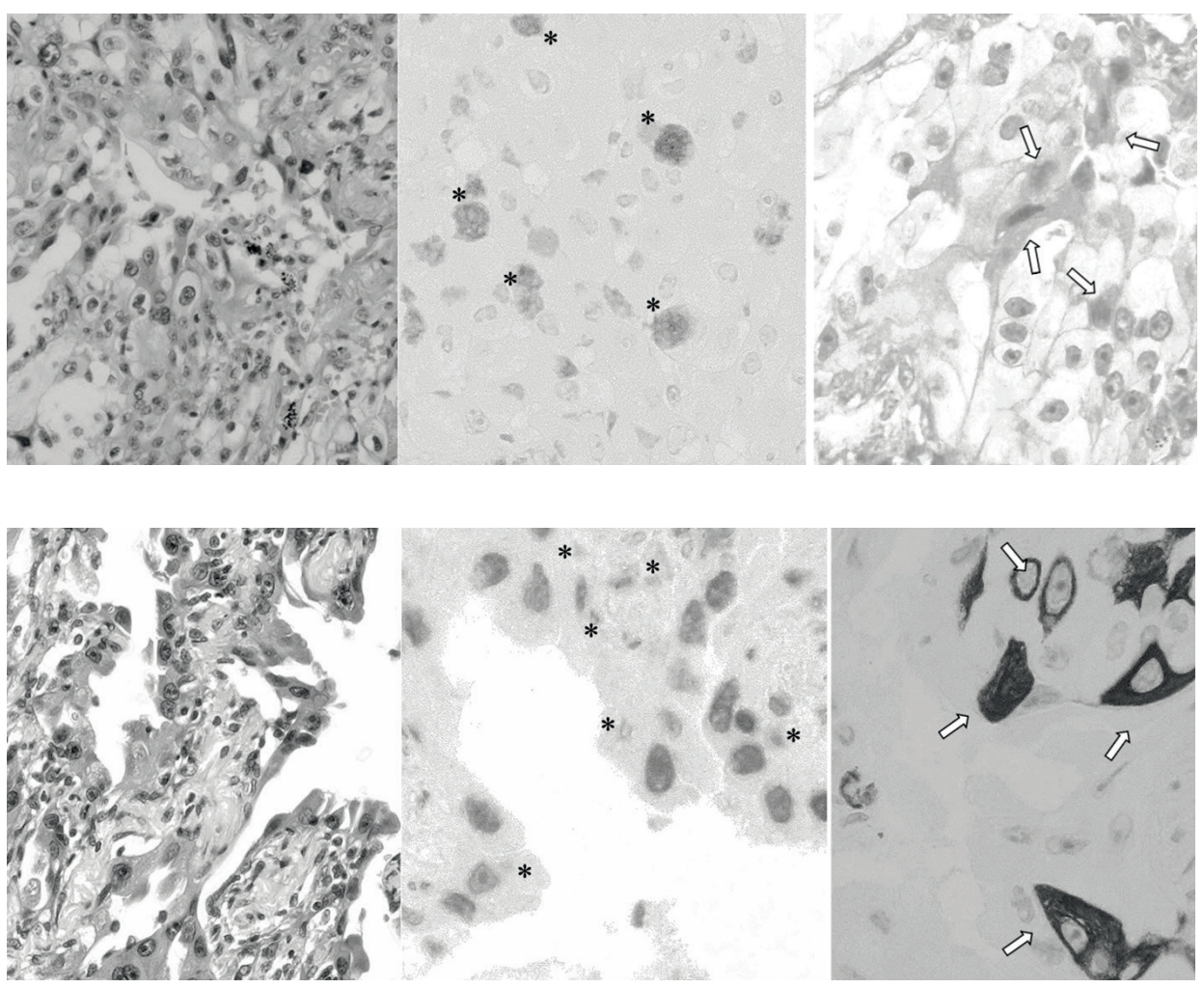

Fig. 2 Histological findings of the resected specimens of the second tumor. The resected tumor showed components of both adenocarcinoma and squamous cell carcinoma, with each comprising at least $10 \%$ of the tumor (Hematoxylin-Eosin staining) (A). There were tumor cells with positive for thyroid transcription factor-1 (B), cytokeratin $5 / 6$ (C).

Fig. 3 Histological findings of the resected specimens of the first tumor. The resected tumor showed components of both adenocarcinoma and squamous cell carcinoma, with each comprising at least $10 \%$ of the tumor (Hematoxylin-Eosin staining)(A). There were tumor cells with positive for Alcian blue (B) and cytokeratin 5/6 (C). 
components with intercellular bridges were $15 \%$ of the tumor (Figure 2-A). There were tumor cells with positive for thyroid transcription factor-1 (Figure 2-B), cytokeratin 5/6 (Figure 2-C), and Alcian Blue (Al-b). An EGFR Exon 21 L858R mutation was identified. Histologically, the nodule was diagnosed as stage IA adenosquamous cell carcinoma with EGFR mutation. At that time, these were evaluated as metachronous double primary tumors because they were different histological types of lung cancer. However, EGFR mutation type in both tumors were the same one, re-examination and comparison of these tumors were performed. Morphologically the cancer cells of the first tumor was very similar to those in the second tumor (Figure 3-A). There were tumor cells with positive for Al-b (Figure 3-B) and cytokeratin 5/6 (Figure $3-C)$. Based on these findings with same EGFR mutation, the second tumor was diagnosed to be a metastasis to the contralateral lung of the first tumor rather than de novo second primary caner. The patient received any additional therapy and is followed up for 3-year period with no recurrence.

\section{DISCUSSION}

Clinical history, radiographic findings and histologic features are most helpful in the differential diagnosis of pulmonary metastasis from the primary lung cancer and the second primary lung cancer. As a feature on CT images of lung metastatic lesions, typical features of hematogenous metastases include single or multiple, round to oval shaped, well-circumscribed radiographic masses without spiculations, calcifications, or tree-in-bud appearance. However, lung lesions in some patients show atypical radiographic features and a possibility of metastasis should be suspected in patients with a history of lung cancer, even if a long time has passed since the diagnosis and treatment of the primary lung cancer $(4,5)$. Kondo et al reported a patient who had a contralateral pleural tumor 14-year after undergoing complete resection of primary lung adenocarcinoma (1). Histologic and genetic analysis of the previously resected specimen and the samples obtained of a newly performed biopsy confirmed that deletion Ex 19 EGFR mutated adenocarcinoma, and the recurrence of lung cancer was determined (1). CT image of the patient was contralateral pleural thickening. Although the pleural thickening had positive positron emission tomography, however, it was evaluated not as an image that required distinguishing between lung metastasis and second primary lung cancer.

Whenever a well-circumscribed lung tumor identified in chest CT scan, the possibility of metastatic lung cancer should be considered and excluded. Genetic studies are at present mandatory for pathologic diagnosis if the primary lung cancer was known. EGFR is one of the most common and important gene mutations. As far as East Asians are concerned, EGFR mutation is expressed in about a half of adenocarcinomas (6-8). However, the positive rate of EGFR mutation in adenosquamous cell carcinoma is reported only as 0 to $5 \%$ (9-13). In our patient, morphologically, both the primary tumor and the second tumor developed contralateral side consisted of the components of adenocarcinoma as well as squamous cell carcinoma, each comprising more than $10 \%$ of the tumor. Therefore, the final pathological diagnosis was lung adenosquamous cell carcinoma. Exon 21 R858R EGFR mutation was identical in both tumors.

It has been evaluated that patients with lung adenosquamous cell carcinoma had poorer prognosis than those with lung adenocarcinoma and those with lung squamous cell carcinoma $(2,3)$. For patients presenting early-staged lung adenosquamous cell carcinoma, surgical removal of the tumor offers patient benefit. EGFR-TKI could be a useful treatment option for EGFR mutated patients with unresectable disease. However, it has not been established yet whether postoperative adjuvant treatment with EGFR-TKI for patients with completely resected lung adenosquamous cell carcinoma such as our patient will affect the prognosis or not.

In our patient, the possibility of "the second cancer" with "different" genetic background cannot be denied, but the possibility of second cancer having the "same" genetic background remains. Secondary cancer had some unclear part with the surroundings and had a pleural indentation as it located adjacent to pleura. But these findings did not suggest that the second tumor was the second cancer having the "same" genetic background. If blood vessels and lymph flow involved in metastasis could be identified, it can be regarded as metastasis, but it is difficult to confirm morphologically if the lesion enlarged. We evaluated that the possibility of pulmonary metastasis was higher than the possibility of second cancer having the "same" genetic background, but we must admit that there is room for discussion. The best approach for second primary lung cancer remains a subject of debate. For resectable second primary lung cancer, surgical intervention is feasible and potentially effective and good prognosis can be expected for these patients (14). On the other hand, if the lesion is assessed as distant metastasis, chemotherapy, a systemic therapy, is indicated for most patients with lung cancer. Their prognosis must be poor. We supposed that treatment strategy can be similar in EGFR gene mutation patients and in negative patients although there is no report that clearly clarified it. In this patient, EGFR-TKI administration might be indicated if it was evaluated to be a recurrence. However, surgical treatment was performed because of resectable oligometastasis after long-term interval. If it was evaluated as a resectable second lung cancer, it was appropriate to perform surgical resection.

To the best of our knowledge, this is the first report of metachronous isolated contralateral lung metastasis from pulmonary adenosquamous carcinoma with EGFR mutation. The patient has been survived with disease free at present for the 84 months following the diagnosis of the first lung adenosquamous carcinoma. It was not possible to differentiate the recurrence from the second lung cancer as a result in our patient, since the driver gene was the same. If we are not sure whether it is recurrence or secondary cancer, it is important to evaluate and compare each tumor resected. We do believe that driver gene evaluation including EGFR mutation may provide useful information in differentiating recurrence from secondary 
cancer in some lung cancer patients. From this perspective, we emphasize the benefits of our experience for real-life practice.

\section{CONFLICT OF INTEREST}

None.

\section{FINANCIAL SUPPORT}

None.

\section{REFERENCES}

1. Kondo N, Takuwa T, Hashimoto M, et al. Gene Mutation Analysis in determining late recurrence of adenocarcinoma of the lung. Ann Thorac Surg 2015; 100(2): 711-3.

2. Filosso PL, Ruffini E, Asioli S, et al. Adenosquamous lung carcinomas: a histologic subtype with poor prognosis. Lung Cancer 2011; 74(1): 25-9.

3. Cooke DT, Nguyen DV, Yang Y, et al. Survival comparison of adenosquamous, squamous cell, and adenocarcinoma of the lung after lobectomy. Ann Thorac Surg 2010; 90(3): 943-8.

4. Miyazaki K, Ano T, Nakazawa K, et al. Late fatal recurrence in gefitinib-treated NSCLC patients. Tuberk Toraks 2007; 55(4): 400-3.
5. Kagohashi K, Nakayama H, Kagei K, et al. Thoracic radiotherapy for mediastinal nodal recurrence. Acta Medica (Hradec Kralove) 2009; $52(1): 23-5$.

6. Jiang $\mathrm{H}$. Overview of gefitinib in non-small cell lung cancer: an Asian perspective. Jpn J Clin Oncol 2009; 39(3): 137-50.

7. Armour AA, Watkins CL. The challenge of targeting EGFR: experience with gefitinib in nonsmall cell lung cancer. Eur Respir Rev 2010; 19(117): 186-96.

8. Dungo RT, Keating GM. Afatinib: first global approval. Drugs 2013; 73(13): 1503-15.

9. Marchetti A, Martella C, Felicioni L, et al. EGFR mutations in nonsmall-cell lung cancer: analysis of a large series of cases and development of a rapid and sensitive method for diagnostic screening with potential implications on pharmacologic treatment. J Clin Oncol 2005; 23(4): 857-65.

10. Sugio K, Uramoto H, Ono K, et al. Mutations within the tyrosine kinase domain of EGFR gene specifically occur in lung adenocarcinoma patients with a low exposure of tobacco smoking. Br J Cancer 2006; 94(6): 896-903.

11. Tsao MS, Sakurada A, Ding K, et al. Prognostic and predictive value of epidermal growth factor receptor tyrosine kinase domain mutation status and gene copy number for adjuvant chemotherapy in nonsmall cell lung cancer. J Thorac Oncol 2011; 6(1): 139-47.

12. Miyamae Y, Shimizu K, Hirato J, et al. Significance of epidermal growth factor receptor gene mutations in squamous cell lung carcinoma. Oncol Rep 2011; 25(4): 921-8.

13. Cancer Genome Atlas Research Network. Comprehensive genomic characterization of squamous cell lung cancers. Nature 2012; 489(7417): 519-25.

14. Sato S, Nakamura M, Shimizu Y, et al. Impact of postoperative complications on outcomes of second surgery for second primary lung cancer. Surg Today 2020 Jun 1. 